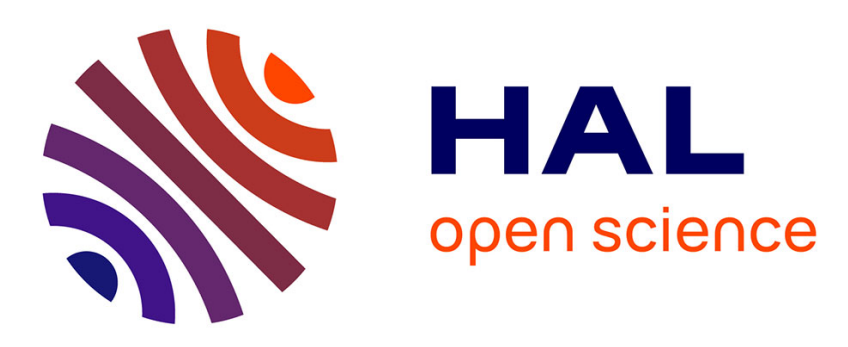

\title{
Variational linear comparison bounds for nonlinear composites with anisotropic phases. II. Crystalline materials
}

\author{
Martin Idiart, Pedro Ponte Castañeda
}

\section{- To cite this version:}

Martin Idiart, Pedro Ponte Castañeda. Variational linear comparison bounds for nonlinear composites with anisotropic phases. II. Crystalline materials. Proceedings of the Royal Society A: Mathematical, Physical and Engineering Sciences, 2007, 463, pp.925-943. 10.1098/rspa.2006.1804 . hal-00311890

\author{
HAL Id: hal-00311890 \\ https://hal.science/hal-00311890
}

Submitted on 28 Aug 2018

HAL is a multi-disciplinary open access archive for the deposit and dissemination of scientific research documents, whether they are published or not. The documents may come from teaching and research institutions in France or abroad, or from public or private research centers.
L'archive ouverte pluridisciplinaire HAL, est destinée au dépôt et à la diffusion de documents scientifiques de niveau recherche, publiés ou non, émanant des établissements d'enseignement et de recherche français ou étrangers, des laboratoires publics ou privés. 


\title{
Variational linear comparison bounds for nonlinear composites with anisotropic phases. II. Crystalline materials
}

\author{
By Martín I. Idiart ${ }^{1,2}$ and Pedro Ponte Castañeda ${ }^{1,2, *}$ \\ ${ }^{1}$ Department of Mechanical Engineering and Applied Mechanics, \\ University of Pennsylvania, Philadelphia, PA 19104-6315, USA \\ ${ }^{2}$ Laboratoire de Mécanique des Solides, C.N.R.S. UMR 7649, Département de \\ Mécanique, École Polytechnique, 91128 Palaiseau Cedex, France
}

In part I of this work, bounds were derived for the effective potentials of nonlinear composites with anisotropic constituents, making use of an appropriate generalization of the linear comparison variational method. In this second part, the special case of nonlinear composites with crystalline constituents is considered. First, it is shown that, for this special but very important class of materials, the 'variational' bounds of part I are at least as good as an earlier version of the bounds due to deBotton \& Ponte Castañeda. Then, the relative merits of these two types of bounds are studied in the context of a model, two-dimensional, porous composite with a power-law crystalline matrix phase, under anti-plane loading conditions. The results show that, indeed, the variational bounds of part I improve, in general, on the earlier bounds, with the former becoming progressively sharper than the latter as the number of slip systems of the crystalline matrix phase increases. In particular, it is shown that, unlike the bounds of deBotton \& Ponte Castañeda, the variational bounds of part I are able to recover the variational bound for composites with an isotropic matrix phase, as the number of slip systems, all having the same flow stress, tends to infinity.

Keywords: nonlinear homogenization; variational methods; crystalline materials

\section{Crystalline phases and polycrystals}

In part I of this work (Idiart \& Ponte Castañeda 2007), bounds have been derived for the effective stress potentials of nonlinear composites made of a fairly general class of anisotropic constituents, satisfying a certain 'square convexity' hypothesis. These bounds were obtained by making use of an appropriate generalization of the linear comparison variational method, introduced by Ponte Castañeda (1991) in the context of composites with isotropic constituents, and will be referred to here as 'variational' bounds. In this second part of the work, we consider the special case of nonlinear composites with crystalline constituents, including polycrystals, which is perhaps the most common type of composite material with anisotropic constituents. It will be shown that the variational

* Author for correspondence (ponte@seas.upenn.edu). 
bounds are at least as good as an earlier version of the bounds due to deBotton \& Ponte Castañeda (1995), which were developed specifically for nonlinear composites with crystalline constituents.

We consider a reference single crystal which is capable of undergoing viscoplastic deformation on a set of $K$ preferred crystallographic slip systems. These systems are characterized by the second-order tensors $\boldsymbol{\mu}_{(k)}, k=1, \ldots, K$, defined by

$$
\boldsymbol{\mu}_{(k)}=\frac{1}{2}\left(\boldsymbol{n}_{(k)} \otimes \boldsymbol{m}_{(k)}+\boldsymbol{m}_{(k)} \otimes \boldsymbol{n}_{(k)}\right),
$$

where $\boldsymbol{n}_{(k)}$ and $\boldsymbol{m}_{(k)}$ are the unit vectors normal to the slip plane and along the slip direction in the $k$ th system, respectively. When the crystal is subjected to an applied stress $\boldsymbol{\sigma}$, the resolved shear stress acting on the $k$ th slip system is given by $\tau_{(k)}=\boldsymbol{\sigma} \cdot \boldsymbol{\mu}_{(k)}$ and the strain (rate) $\boldsymbol{\varepsilon}$ in the crystal is the superposition of the strain (rates) $\gamma_{(k)}$ on each slip system $k(k=1, \ldots, K)$. They are assumed to depend on the resolved shear stress $\tau_{(k)}$, through a slip potential $\psi_{(k)}$, such that $\gamma_{(k)}=\psi_{(k)}^{\prime}\left(\tau_{(k)}\right)$. For consistency with the hypothesis of square convexity introduced in part I, the potentials $\psi_{(k)}$ will be assumed here to be convex in the variable $\tau_{(k)}^{2}$ (and are therefore also convex in $\tau_{(k)}$ ). A commonly used form for the slip potentials $\psi_{(k)}$ is the power-law form

$$
\psi_{(k)}(\tau)=\frac{\gamma_{0}\left(\tau_{0}\right)_{(k)}}{n+1}\left(\frac{|\tau|}{\left(\tau_{0}\right)_{(k)}}\right)^{n+1},
$$

where $m=1 / n(0 \leq m \leq 1)$ and $\left(\tau_{0}\right)_{(k)}$ are the strain-rate sensitivity and flow stress of the $k$ th slip system, respectively, and $\gamma_{0}$ is a reference strain rate. Note that the limiting values of the exponent $m=1$ and 0 correspond to linear and rigid-ideally plastic behaviours, respectively. In this connection, it is recalled that, even though the slip potentials $\psi_{(k)}$ are not differentiable in the rigid-ideally plastic case, it is still possible to relate $\gamma_{(k)}$ and $\tau_{(k)}$ via the subdifferential of convex analysis.

Since the phases in a composite made of such crystalline materials may also exhibit different orientations, it is useful to introduce a set of rotation tensors $\mathbf{R}^{(r)}$ $(r=1 \ldots, N)$. Then, defining phase $r$ as the region occupied by all crystals of a given type and orientation $\mathbf{R}^{(r)}$, its constitutive behaviour is characterized by the stress potential

$$
u^{(r)}(\boldsymbol{\sigma})=\sum_{k=1}^{K^{(r)}} \psi_{(k)}^{(r)}\left(\tau_{(k)}^{(r)}\right)
$$

where the functions $\psi_{(k)}^{(r)}$ characterize the constitutive response of the slip systems of all the crystals associated with phase $r$ and

$$
\tau_{(k)}^{(r)}=\boldsymbol{\sigma} \cdot\left(\mathbf{R}^{(r)}{ }^{T} \boldsymbol{\mu}_{(k)}^{(r)} \mathbf{R}^{(r)}\right) .
$$

It is recalled that a polycrystal is an aggregate of a large number of identical single crystals with different orientations. This special case is included in expression (1.3), provided that all the phase slip systems and potentials be taken identical to each other $\left(\boldsymbol{\mu}_{(k)}^{(r)}=\boldsymbol{\mu}_{(k)}\right.$ and $\left.\psi_{(k)}^{(r)}=\psi_{(k)}\right)$. But the definition (1.3) is general enough to include multi-phase polycrystals, as well as composites with crystalline phases, such as the porous crystalline materials considered in $\$ 1 a$. 


\section{(a) Variational bounds}

Since the phase stress potentials (1.3) have been assumed to be 'square convex', the variational bound given in result 4.4 of part I holds and can be used for the above-defined class of composites with crystalline phases.

Result 1.1. The effective stress potential $\widetilde{u}$ of an $N$-phase nonlinear composite (or polycrystal) with crystalline phase potentials (1.3) is bounded below by

$$
\tilde{u}_{-}(\overline{\boldsymbol{\sigma}})=\sup _{\substack{\mathbb{S}_{0}^{(r)} \geq 0 \\ r=1, \ldots, N}}\left\{\tilde{u}_{0}(\overline{\boldsymbol{\sigma}})-\sum_{r=1}^{N} c^{(r)} v^{(r)}\left(\mathbb{S}_{0}^{(r)}\right)\right\},
$$

where the error functions $v^{(r)}$ are given by

and where

$$
v^{(r)}\left(\mathbb{S}_{0}^{(r)}\right)=\sup _{\boldsymbol{\sigma}}\left\{\frac{1}{2} \boldsymbol{\sigma} \cdot \mathbb{S}^{(r)} \boldsymbol{\sigma}-u^{(r)}(\boldsymbol{\sigma})\right\},
$$

$$
\tilde{u}_{0}(\overline{\boldsymbol{\sigma}})=\frac{1}{2} \overline{\boldsymbol{\sigma}} \cdot \tilde{\mathbb{S}}_{0}\left(\mathbb{S}_{0}^{(s)}\right) \overline{\boldsymbol{\sigma}}
$$

is the effective stress potential of a linear comparison composite (LCC) with uniform compliance tensors $\mathbb{S}_{0}^{(r)}$ in each of the phases $(r=1 \ldots, N)$, and effective compliance tensor $\tilde{\mathbb{S}}_{0}$

As discussed in remark 4.5 of part I, the bound (1.5) involves a non-smooth, concave optimization problem for the variables $\mathbb{S}_{0}^{(r)}$, which can be solved by making use of appropriate numerical methods. However, as will be seen in the context of the model problem considered below, the main difficulty in the determination of this bound lies in the computation of the 'error' functions $v^{(r)}$, which involve a non-concave optimization problem that must be solved making use of more sensitive numerical algorithms.

If the slip potentials $\psi_{(k)}^{(r)}$ are all of the power-law type (1.2) with the same exponent $n$, as in the model problem considered in $\$ 2$, the bound (1.5) admits the following alternative representation, as can be deduced from result 4.6 of part I.

Result 1.2. The effective stress potential $\widetilde{u}$ of an $N$-phase, power-law composite (or polycrystal) with crystalline phase potentials given by equation (1.3), together with equation (1.2), is bounded below by

$$
\tilde{u}_{-}(\overline{\boldsymbol{\sigma}})=\frac{2}{n+1} \sup _{\substack{\mathbb{S}_{0}^{(r)} \geq 0 \\ r=1, \ldots, N}}\left\{\tilde{u}_{0}(\overline{\boldsymbol{\sigma}})^{(n+1) / 2}\left[\frac{n+1}{n-1} \sum_{r=1}^{N} c^{(r)} v^{(r)}\left(\mathbb{S}_{0}^{(r)}\right)\right]^{(1-n) / 2}\right\},
$$

where $\tilde{u}_{0}$ is given by (1.7), and

$$
v^{(r)}\left(\mathbb{S}_{0}\right)=\frac{1}{2} \frac{n-1}{n+1} \sup _{\|\boldsymbol{\sigma}\|=1}\left\{\frac{\left[\boldsymbol{\sigma} \cdot \mathbb{S}_{0} \boldsymbol{\sigma}\right]^{(n+1) /(n-1)}}{\left[(n+1) u^{(r)}(\boldsymbol{\sigma})\right]^{2 /(n-1)}}\right\} .
$$

The main advantage of this special representation for power-law composites is that, even though the functions $v^{(r)}$ as given by equation (1.9) still require the 
solution of a non-concave optimization problem, this optimization is now over a bounded domain $(\|\boldsymbol{\sigma}\|=1)$. In addition, expression (1.8) is well behaved for large values of the nonlinearity $n$, which facilitates the numerical resolution of the problem. In general, further simplifications are not possible for potentials $u^{(r)}$ of the form (1.3), and the solution strategies must be adapted to the specific system of interest.

However, considerable simplification is possible for ideally plastic composites. It is then convenient to introduce the strength domain $P^{(r)}$ of each phase $r$, defined by the conditions $u^{(r)}(\boldsymbol{\sigma})=0$ if $\boldsymbol{\sigma} \in P^{(r)}$, and $\infty$ otherwise. For crystalline phases characterized by ideally plastic slip potentials of the form (1.2) with $n \rightarrow \infty$, the sets $P^{(r)}$ are polyhedral; therefore, the extreme points of those sets are given by their finitely many vertices (see Rockafellar 1970). This fact allows the following, further specialization of result 4.7 of part I, for the effective strength domain $\tilde{P}$ of an ideally plastic composite with crystalline phases.

Result 1.3. The effective strength domain $\widetilde{P}$ of an $N$-phase composite (or polycrystal) with crystalline, rigid-ideally plastic phases is bounded from the outside by

$$
\tilde{P}_{+}=\left\{\overline{\boldsymbol{\sigma}} \mid \tilde{u}_{0}(\overline{\boldsymbol{\sigma}}) \leq \sum_{r=1}^{N} c^{(r)} v^{(r)}\left(\mathbb{S}_{0}^{(r)}\right), \quad \forall \mathbb{S}_{0}^{(r)} \geq 0\right\},
$$

where $\tilde{u}_{0}$ is given by equation (1.7), and the functions $v^{(r)}$ are given by

$$
v^{(r)}\left(\mathbb{S}_{0}^{(r)}\right)=\max _{k=1, \ldots, K_{\mathrm{v}}^{(r)}}\left\{\frac{1}{2} \boldsymbol{\sigma}_{(k)}^{(r)} \cdot \mathbb{S}_{0}^{(r)} \boldsymbol{\sigma}_{(k)}^{(r)}\right\},
$$

where $K_{\mathrm{v}}^{(r)}$ is the total number of vertices of $P^{(r)}$, and $\boldsymbol{\sigma}_{(k)}^{(r)}$ denotes the stress vector associated with the kth vertex.

Thus, in the case of ideally plastic, crystalline phases, evaluation of the functions $v^{(r)}$ as given by equation (1.11) is very simple, requiring only knowledge of the vertex stress vectors $\boldsymbol{\sigma}_{(k)}$ of the crystal in question. Such geometric information on the yield surface is already available for common crystal symmetries, since it is required in applications of the classical Taylor theory of polycrystal plasticity.

\section{(b) Relaxed variational bounds}

As already mentioned, there is an alternative version of the variational bound introduced by deBotton \& Ponte Castañeda (1995) for composites with crystalline phase potentials of the form (1.3). With the objective of establishing a relationship between the new bound (1.5) and this earlier version, the rest of this section is devoted to a derivation of the bound of deBotton \& Ponte Castañeda (1995) directly from equation (1.5).

Let the compliance tensors $\mathbb{S}_{0}^{(r)}$ in equation (1.5) take the special form

$$
\mathbb{S}_{0}^{(r)}=2 \sum_{k=1}^{K^{(r)}} \alpha_{(k)}^{(r)} \boldsymbol{\mu}_{(k)}^{(r)} \otimes \boldsymbol{\mu}_{(k)}^{(r)}, \quad \alpha_{(k)}^{(r)} \geq 0,
$$


where the $\boldsymbol{\mu}_{(k)}^{(r)}$ are those of the nonlinear crystalline phase $r$. Then, recalling the definition (1.6) of the functions $v^{(r)}$, it follows that

$$
\begin{aligned}
& v^{(r)}\left(\mathbb{S}_{0}^{(r)}\right)=\sup _{\boldsymbol{\sigma}}\left\{\frac{1}{2} \boldsymbol{\sigma} \cdot \mathbb{S}_{0}^{(r)} \boldsymbol{\sigma}-u^{(r)}(\boldsymbol{\sigma})\right\} \\
& =\sup _{\boldsymbol{\sigma}} \sum_{k=1}^{K^{(r)}}\left\{\alpha_{(k)}^{(r)}\left(\sigma \cdot \boldsymbol{\mu}_{(k)}^{(r)}\right)^{2}-\psi_{(k)}^{(r)}\left(\sigma \cdot \boldsymbol{\mu}_{(k)}^{(r)}\right)\right\} \\
& \leq \sum_{k=1}^{K^{(r)}} \sup _{(k)}^{(r)}\left\{\alpha_{(k)}^{(r)}\left(\tau_{(k)}^{(r)}\right)^{2}-\psi_{(k)}^{(r)}\left(\tau_{(k)}^{(r)}\right)\right\}
\end{aligned}
$$

and therefore

$$
v^{(r)}\left(\mathbb{S}_{0}^{(r)}\right) \leq \sum_{k=1}^{K^{(r)}} v_{(k)}^{(r)}\left(\alpha_{(k)}^{(r)}\right),
$$

where

$$
v_{(k)}^{(r)}\left(\alpha_{(k)}^{(r)}\right)=\sup _{\tau}\left\{\alpha_{(k)}^{(r)} \tau^{2}-\psi_{(k)}^{(r)}(\tau)\right\} .
$$

In view of the square convexity hypothesis for the slip potentials $\psi_{(k)}^{(r)}$, the expression inside the curly brackets in equation (1.15) is concave in $\tau^{2}$, and so the computation of the functions $v_{(k)}^{(r)}$ is straightforward, as opposed to that of the functions $v^{(r)}$, which as already stated requires, in general, the solution of a nonconcave optimization problem. For this reason, the bound of deBotton \& Ponte Castañeda (1995), which follows from equation (1.14) and is detailed in the next result, is much simpler to compute.

Result 1.4. The lower bound (1.5) for the effective stress potential $\widetilde{u}$ of an $N$-phase nonlinear composite with crystalline phase potentials (1.3) is bounded below by

$$
\tilde{u}_{R-}(\overline{\boldsymbol{\sigma}})=\sup _{\substack{\alpha_{(k)}^{(r)} \geq 0 \\ r=1, \ldots, N \\ k=1, \ldots, K^{(r)}}}\left\{\tilde{u}_{0}(\overline{\boldsymbol{\sigma}})-\sum_{r=1}^{N} \sum_{k=1}^{K^{(r)}} c^{(r)} v_{(k)}^{(r)}\left(\alpha_{(k)}^{(r)}\right)\right\},
$$

where $\tilde{u}_{0}$ is the effective stress potential of an LCC, defined by equation (1.7), with phase compliance tensors $\mathbb{S}_{0}^{(s)}$, as given by equation (1.12) in terms of the slip compliances $\alpha_{(k)}^{(s)}$, and the functions $v_{(k)}^{(r)}$ are determined by relations (1.15).

Owing to its derivation here, the bound equation (1.16) will be referred to as the 'relaxed variational' (linear comparison) bound, as opposed to the bound (1.5), which will be plainly called the variational (linear comparison) bound.

For power-law and ideally plastic crystalline phases, the functions $v_{(k)}^{(r)}$ can be computed explicitly and the 'relaxed' bounds can be simplified further. The results are quoted below (Ponte Castañeda \& Suquet 1998) for completeness. 
Result 1.5. For N-phase composites with power-law crystalline phases, the relaxed variational bound (1.16) can be rewritten as

$$
\begin{aligned}
& \tilde{u}_{R-}(\overline{\boldsymbol{\sigma}})=\frac{\gamma_{0}}{n+1} \quad \sup _{\alpha_{(k)}^{(r)} \geq 0} \\
& r=1, \ldots, N \text {, } \\
& k=1, \ldots, K^{(r)} \\
& \left\{\left[\tilde{u}_{0}(\overline{\boldsymbol{\sigma}})\right]^{(n+1) / 2}\left[\sum_{r=1}^{N} \sum_{k=1}^{K^{(r)}} c^{(r)}\left(\alpha_{(k)}^{(r)}\right)^{(1+n) /(n-1)}\left(\left(\tau_{0}\right)_{(k)}^{(r)}\right)^{2 n /(n-1)}\right]^{(1-n) / 2}\right\} .
\end{aligned}
$$

Also, the effective strength domain $\widetilde{P}$ of an $N$-phase composite with rigid-ideally plastic $(n \rightarrow \infty)$, crystalline phases is bounded from the outside by

$$
\tilde{P}_{R+}=\left\{\overline{\boldsymbol{\sigma}} \mid \tilde{u}_{0}(\overline{\boldsymbol{\sigma}}) \leq \sum_{r=1}^{N} \sum_{k=1}^{K^{(r)}} c^{(r)} \alpha_{(k)}^{(r)}\left(\left(\tau_{0}\right)_{(k)}^{(r)}\right)^{2}, \quad \forall \alpha_{(k)}^{(r)} \geq 0\right\} .
$$

It is worth noting that equality in equation (1.14) holds if the set of $K^{(r)}$ tensors $\boldsymbol{\mu}_{(k)}^{(r)}$ form a basis for the space of stress tensors, since in that case, the scalar quantities $\tau_{(k)}^{(r)}$ represent the components of a stress tensor relative to that basis. This suggests that the relaxed variational bound (1.16) coincides with the variational bound (1.5) when this is the case, and the optimal $\hat{\mathbb{S}}_{0}^{(r)}$ in the context of the latter are of the form (1.12). This is precisely what happens in the model problem to be discussed below. Unfortunately, it is not representative of what happens in practice, since for most cases, including FCC, BCC and HCP crystals, the number of available slip systems is larger than the dimension of the relevant stress space.

\section{Application to porous crystalline materials}

In this section, the focus is on a special class of (two-phase) porous materials with 'particulate' microstructures, consisting of aligned cylindrical pores $(r=2)$ that are distributed randomly and isotropically in a viscoplastic single-crystal phase $(r=1)$. It is assumed that the symmetry axes of the crystalline matrix and the cylindrical pores are aligned with the $x_{3}$ axis. It is further assumed that the behaviour of the crystalline matrix is characterized by an incompressible stress potential $u^{(1)}$ of the form (1.3), where the slip potentials $\psi_{(k)}^{(1)}$ are of the powerlaw type (1.2), and the Schmid tensors $\boldsymbol{\mu}_{(k)}$ are taken to be of the form

$$
\boldsymbol{\mu}_{(k)}=\frac{1}{2}\left(\boldsymbol{n}_{(k)} \otimes \boldsymbol{e}_{3}+\boldsymbol{e}_{3} \otimes \boldsymbol{n}_{(k)}\right) .
$$

Here, $e_{3}$ is parallel to the slip direction and

$$
\boldsymbol{n}_{(k)}=\cos \theta_{(k)} \boldsymbol{e}_{1}+\sin \theta_{(k)} \boldsymbol{e}_{2},
$$

denotes the unit vector normal to the slip plane of the $k$ th system, relative to a laboratory frame of reference $\boldsymbol{e}_{i}$ (in the sequel, components are always referred to this basis). The porous material is subjected to anti-plane loadings, and the 

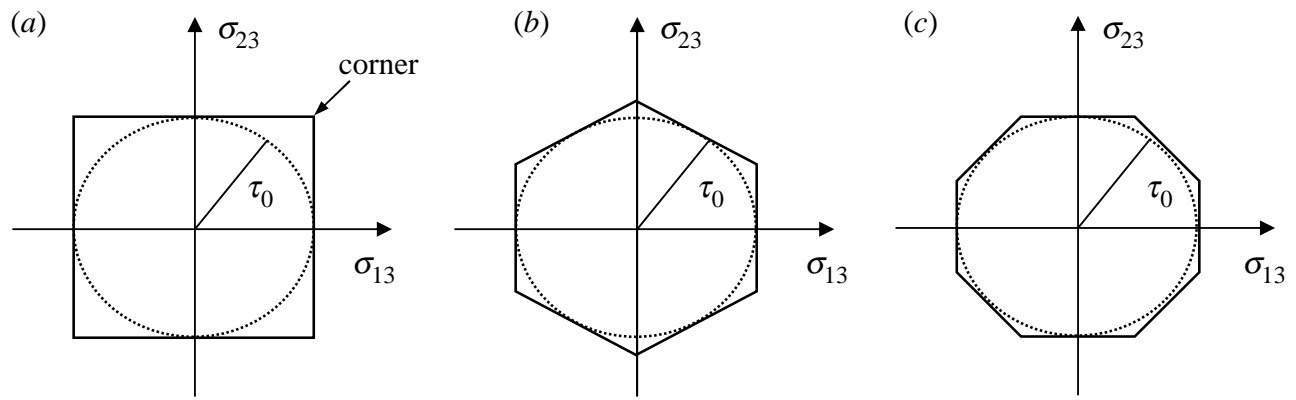

Figure 1. Yield surfaces in $\boldsymbol{\sigma}_{13}-\boldsymbol{\sigma}_{23}$ space for $(a)$ square $(K=2),(b)$ hexagonal $(K=3)$ and (c) octagonal $(K=4)$ symmetries.

relevant viscoplastic boundary value problem becomes a vectorial two-dimensional problem, where the non-zero components of the stress and strain-rate vectors, namely, $\boldsymbol{\sigma}_{13}, \boldsymbol{\sigma}_{23}, \boldsymbol{\varepsilon}_{13}$ and $\boldsymbol{\varepsilon}_{23}$, are functions of $x_{1}$ and $x_{2}$ only. (This problem is mathematically equivalent to two-dimensional conductivity.)

For simplicity, it will be assumed initially that all slip systems have the same flow stress, i.e., $\left(\tau_{0}\right)_{(k)}=\tau_{0}$ for all $k$. Of particular interest here are three different types of anisotropy, characterized by the sets of angles $\theta_{(k)}$ given by $\{0, \pi / 2\}$, $\{0, \pm \pi / 3\}$ and $\{0, \pm \pi / 4, \pi / 2\}$, which correspond to square $(K=2)$, hexagonal $(K=3)$ and octagonal $(K=4)$ symmetry, respectively. In the linear case, the potential $u^{(1)}$ is in fact isotropic for these three types of anisotropies. In the nonlinear case, however, the potential $u^{(1)}$ is, in general, anisotropic and in the ideally plastic limit, it defines an anisotropic polygonal yield surface in the $\sigma_{13}-\sigma_{23}$ stress space, as depicted in figure 1 . Note that as the number of slip systems $K$ increases, the potential approaches an isotropic yield surface with flow stress $\tau_{0}$.

From the homogeneity of the potential (1.3) and the symmetry of the problem, it follows that, under anti-plane conditions, the effective stress potential can be written as

$$
\tilde{u}(\overline{\boldsymbol{\sigma}})=\frac{\tilde{\tau}_{0} \gamma_{0}}{1+n}\left(\frac{\bar{\tau}_{\mathrm{e}}}{\tilde{\tau}_{0}}\right)^{1+n}
$$

where $\overline{\boldsymbol{\tau}}_{\mathrm{e}}=\sqrt{(1 / 2) \overline{\boldsymbol{\sigma}}_{d} \cdot \overline{\boldsymbol{\sigma}}_{d}}=\left(\bar{\sigma}_{13}^{2}+\bar{\sigma}_{23}^{2}\right)^{1 / 2}$ is the macroscopic equivalent stress and $\tilde{\tau}_{0}$ is the effective flow stress, which depends on the porosity $f=c^{(2)}$ and the direction of loading $\bar{\theta}=\tan ^{-1}\left(\bar{\sigma}_{23} / \bar{\sigma}_{13}\right)$, and completely characterizes the effective response of the porous material. It is noted that, for the particular class of composites considered here, the potential $\tilde{u}$ exhibits the same symmetries as the matrix potential $u^{(1)}$, and therefore $\tilde{\tau}_{0}$ is a periodic function of $\bar{\theta}$ with period $\pi / K$. Thus, it suffices to restrict attention to loading directions in the range $|\bar{\theta}| \leq \pi /(2 K)$. Note that the values $\bar{\theta}=0$ and $\pm \pi /(2 K)$ correspond to loadings directed along a slip system and a 'corner' of the matrix phase, respectively.

\section{(a) Variational bounds}

In this section, the variational bounds described in result 1.2 are specialized to the model porous material introduced above. Thus, from the lower bound (1.8) for the effective stress potential of a power-law composite, we obtain the 
following upper bound for the effective flow stress of the model porous material:

$$
\tilde{\tau}_{0}(\bar{\theta})=\left(\frac{\gamma_{0}}{2}\right)^{1 / n} \inf _{\mathbb{S}_{0}^{(1)} \geq 0}\left\{\left[\tilde{u}_{0}\left(\overline{\boldsymbol{\sigma}} / \bar{\tau}_{\mathrm{e}}\right)\right]^{-(n+1) / 2 n}\left[(1-f) \frac{n+1}{n-1} v^{(1)}\left(\mathbb{S}_{0}^{(1)}\right)\right]^{(n-1) / 2 n}\right\},
$$

where $\tilde{u}_{0}$ and $v^{(1)}$ are defined by equations (1.6) and (1.7), respectively.

For the problem at hand, it suffices to restrict the optimization over the compliance tensors $\mathbb{S}_{0}^{(1)}$ to the out-of-plane components $S_{0(i 3)(j 3)}^{(1)}, i, j=1,2$. It is then convenient to write these tensors in the spectral form

$$
\mathbb{S}_{0}^{(1)}=\frac{1}{2 \lambda_{0}} \mathbb{E}+\frac{1}{2 \mu_{0}} \mathbb{F}
$$

where $\mathbb{E}$ and $\mathbb{F}$ are eigentensors given by $\mathbb{E}=\boldsymbol{\mu}_{0} \otimes \boldsymbol{\mu}_{0}$ and $\mathbb{F}=\mathbb{K}-\mathbb{E}$, with $\boldsymbol{\mu}_{0}=\frac{1}{\sqrt{2}}$ $\left(\boldsymbol{n} \otimes \boldsymbol{e}_{3}+\boldsymbol{e}_{3} \otimes \boldsymbol{n}\right), \quad \boldsymbol{n}=\cos \beta \boldsymbol{e}_{1}+\sin \beta \boldsymbol{e}_{2}, \quad$ and $\mathbb{K}$ denoting the out-of-plane projection of the fourth-order identity tensor. Thus, the tensors $\mathbb{S}_{0}^{(1)}$ are completely specified by two positive moduli $\lambda_{0}$ and $\mu_{0}$ (eigenvalues), and the orientation of their principal axes $\beta$, given by $\tan 2 \beta=2 S_{01323}^{(1)} /\left(S_{01313}^{(1)}-S_{02323}^{(1)}\right)$. Note that the degree of anisotropy of these compliance tensors is characterized by the ratio $k_{0}=\lambda_{0} / \mu_{0}$, which takes the value 1 for isotropic tensors, and 0 or infinity, for strongly anisotropic tensors.

The variational bounds (2.4) require the use of appropriate bounds for the effective potential $\tilde{u}_{0}$ of linearly viscous, porous materials with matrix compliances of the form (2.5). In this work, use is made of the generalized Hashin-Shtrikman (HS) bounds of Willis (1977), which are known to be optimal for (two-phase) porous materials. In this case, they can be expressed in the form

$$
\tilde{\mathbb{S}}_{0}=\mathbb{S}_{0}^{(1)}+\frac{f}{1-f}\left(\mathbb{Q}^{(1)}\right)^{-1},
$$

where $\mathbb{Q}^{(1)}$ is given by $\mathbb{Q}^{(1)}=\mathbb{C}_{0}^{(1)}-\mathbb{C}_{0}^{(1)} \mathbb{P}^{(1)} \mathbb{C}_{0}^{(1)}$. Here, $\mathbb{C}_{0}^{(1)}=\mathbb{S}_{0}^{(1)^{-1}}$, and $\mathbb{P}^{(1)}$ is a microstructural tensor, that can be found in Ponte Castañeda \& Nebozhyn (1997). The resulting effective compliance tensor $\tilde{\mathbb{S}}_{0}$ has the same form as the compliance tensor of the matrix phase (2.5), but with effective moduli given by

$$
\tilde{\lambda}_{0}=\frac{1-f}{1+f \sqrt{k_{0}}} \lambda_{0}, \quad \tilde{\mu}_{0}=\frac{1-f}{1+f / \sqrt{k_{0}}} \mu_{0} .
$$

Note that the compliance tensors $\tilde{\mathbb{S}}_{0}$ and $\mathbb{S}_{0}^{(1)}$ are therefore co-axial, i.e. they have the same principal directions, which is a consequence of the assumed isotropy of the microstructure.

In addition, the bound (2.4) requires the evaluation of the error function $v^{(1)}$. This actually constitutes the main difficulty in the computation of the variational bound, for the optimization with respect to $\tilde{\boldsymbol{\sigma}}^{(1)}$ in equation (1.6) is a non-convex global optimization problem. In general, this optimization has to be carried out numerically, using, for instance, methods based on genetic algorithms, such as the differential evolution algorithm of Storn \& Price (1997). In this connection, it should be emphasized that such optimization methods cannot, in general, guarantee that the result obtained is indeed the global optimum. However, in the context of the model problem considered here, the optimization in equation (1.6) involves only one variable in a bounded domain, namely the orientation angle of the stress vector $\check{\boldsymbol{\sigma}}^{(1)}$, and is therefore a fairly simple one for these methods, 
which have been developed to deal with high-dimensional optimizations. Finally, the optimization of the bound (2.4) with respect to the compliance tensor $\mathbb{S}_{0}^{(1)}$ should be carried out numerically, in general, making use of an appropriate method for non-smooth optimization, since the error function $v^{(1)}$ is not differentiable in its entire domain (see part I).

\section{(i) Rigid-ideally plastic matrix}

It has been shown that the computation of the variational bound simplifies considerably in the case of ideal plasticity. Indeed, in this case, the variational bounds for the model problem considered here can be computed analytically. First, note that in the ideally plastic limit $(n \rightarrow \infty)$, the variational bound (2.4) for $\tilde{\tau}_{0}$ simplifies to

$$
\tilde{\tau}_{0}(\bar{\theta})=\inf _{\mathbb{S}_{0}^{(1)} \geq 0}\left\{\frac{\left.\tilde{u}_{0}(\bar{\sigma}) / \bar{\tau}_{\mathrm{e}}\right)}{(1-f) v^{(1)}\left(\mathbb{S}_{0}^{(1)}\right)}\right\}^{-1 / 2},
$$

which defines a bound from the outside for the macroscopic yield surface of the ideally plastic porous material. The most important simplification, however, comes from the fact that, in this case, the function $v^{(1)}$ can be written in the form (1.11), with $\boldsymbol{\sigma}_{(k)}^{(1)}$ being the stress vectors corresponding to the $2 K$ vertices, or corners, of the matrix yield surface (figure 1). Then, the following explicit expressions for $v^{(1)}$ may be derived by routine analysis:

$$
\begin{aligned}
K=2: v^{(1)}\left(\mathbb{S}_{0}\right)= & \left(\tau_{0}^{2} / 2\right)\left\{\lambda_{0}^{-1}+\mu_{0}^{-1}+\left|\lambda_{0}^{-1}-\mu_{0}^{-1}\right| \sin (|2 \beta|)\right\}, \\
K=3: \quad v^{(1)}\left(\mathbb{S}_{0}\right)= & \left(\tau_{0}^{2} / 3\right)\left\{\lambda_{0}^{-1}+\mu_{0}^{-1}+\left|\lambda_{0}^{-1}-\mu_{0}^{-1}\right| \cos \left[|2 \beta|-\frac{\pi}{3} H\left(1-k_{0}\right)\right]\right\}, \\
K=4: v^{(1)}\left(\mathbb{S}_{0}\right)= & \left(\tau_{0}^{2} / 4\right) \sec ^{2}(\pi / 8)\left\{\lambda_{0}^{-1}+\mu_{0}^{-1}+\cdots\right. \\
& \left.+\left|\lambda_{0}^{-1}-\mu_{0}^{-1}\right| \cos \left[|2 \beta|-\frac{\pi}{4}+\pi H\left(k_{0}-1\right)\right]\right\},
\end{aligned}
$$

where $H$ denotes the Heaviside function, and it is recalled that $k_{0}=\lambda_{0} / \mu_{0}$. These expressions are valid for $|\beta| \leq \pi /(2 K)$, but use can be made of the fact that the functions $v^{(1)}$ are even and periodic in $\beta$, with period $\pi / K$, to obtain corresponding expressions that are valid outside this range. It is emphasized that these functions are differentiable everywhere except at $\beta=0$ and $\lambda_{0}=\mu_{0}$.

The optimization with respect to $\mathbb{S}_{0}^{(1)}$ in equation $(2.8)$ can then be carried out analytically, leading to the following explicit bound for the effective flow stress:

$$
\tilde{\tau}_{0}(\bar{\theta})=\tau_{0} \sec \theta_{\mathrm{c}}(1-f)\left[1+\frac{f}{2} \frac{1+k_{0}}{\sqrt{k_{0}}}\right]^{-1 / 2},
$$

where $\theta_{\mathrm{c}}=\pi /(2 K)$, and the anisotropy ratio $k_{0}$ is a periodic function of $\bar{\theta}$, with period $2 \theta_{\mathrm{c}}$, given by

$$
\sqrt{k_{0}}= \begin{cases}\frac{\sqrt{\left(\cos 2 \bar{\theta}-\cos 2 \theta_{\mathrm{c}}\right)^{2}+f^{2} \sin ^{2} 2 \theta_{\mathrm{c}}}-\left(\cos 2 \bar{\theta}-\cos 2 \theta_{\mathrm{c}}\right)}{\left(1-\cos 2 \theta_{\mathrm{c}}\right) f} & |\bar{\theta}|<\bar{\theta}_{\mathrm{c}}, \\ 1 & \bar{\theta}_{\mathrm{c}} \leq|\bar{\theta}| \leq \theta_{\mathrm{c}}\end{cases}
$$


with the angle $\bar{\theta}_{\mathrm{c}}$ given by $\cos 2 \bar{\theta}_{\mathrm{c}}=\min \left\{(1+f) \cos 2 \theta_{\mathrm{c}}, 1\right\}$. It is noted that, even though $k_{0}$ is not smooth at $\bar{\theta}=\bar{\theta}_{\mathrm{c}}$, the effective flow stress (2.9) turns out to be a smooth function of $\bar{\theta}$.

\section{(b) Relaxed variational bounds}

In this section, the relaxed variational bounds of deBotton \& Ponte Castañeda (1995) recalled in result 1.5 are specialized for the above-described model problem. Thus, from the lower bound (1.17) for the effective stress potential, we obtain the following upper bound for the effective flow stress of the model porous material

$$
\tilde{\tau}_{0}(\bar{\theta})=\tau_{0} \inf _{\alpha_{(k)} \geq 0}\left\{\left[\tilde{u}_{0}\left(\bar{\sigma} / \bar{\tau}_{\mathrm{e}}\right)\right]^{-(n+1) / 2 n}\left[(1-f) \sum_{k=1}^{K}\left(\alpha_{(k)}\right)^{(n+1) /(n-1)}\right]^{(n-1) / 2 n}\right\},
$$

where $\tilde{u}_{0}$ is the effective potential of a linear porous material with matrix compliance tensor

$$
\mathbb{S}_{0}^{(1)}=2 \sum_{k=1}^{K} \alpha_{(k)} \boldsymbol{\mu}_{(k)} \otimes \boldsymbol{\mu}_{(k)},
$$

with the $\boldsymbol{\mu}_{(k)}$ given by equation (2.1). The tensor (2.12) can be written in the form (2.5), where the tensors $\mathbb{E}$ and $\mathbb{F}$ depend on the orientation of the principal axes of equation (2.12), $\beta$, and the moduli $\lambda_{0}$ and $\mu_{0}$ are given by

$$
\frac{1}{2 \lambda_{0}}=2 \sum_{k=1}^{K} \alpha_{(k)}\left(\boldsymbol{\mu}_{(k)} \cdot \mathbb{E} \boldsymbol{\mu}_{(k)}\right), \quad \frac{1}{2 \mu_{0}}=2 \sum_{k=1}^{K} \alpha_{(k)}\left(\boldsymbol{\mu}_{(k)} \cdot \mathbb{F} \boldsymbol{\mu}_{(k)}\right) .
$$

Then, the linear Hashin-Shtrikman bounds for the effective stress potential $\tilde{u}_{0}$ of the LCC in equation (2.11) are given by expressions (2.6) and (2.7), with $\lambda_{0}, \mu_{0}, \mathbb{E}$ and $\mathbb{F}$ being those associated with the compliance tensor (2.12).

Finally, the optimization with respect to the variables $\alpha_{(k)}$ in equation (2.11) should be carried out numerically using a smooth optimization method.

\section{(i) Ideally plastic matrix}

In the ideally plastic limit, the relaxed variational bound (2.11) for $\tilde{\tau}_{0}$ can be shown to reduce to

$$
\tilde{\tau}_{0}(\bar{\theta})=\sqrt{2} \tau_{0}(1-f) \inf _{\alpha_{(k)} \geq 0}\left\{1+\frac{1-k_{0}}{1+k_{0}} \cos [2(\bar{\theta}-\beta)]+\frac{2 \sqrt{k_{0}}}{1+k_{0}} f\right\}^{-1 / 2} .
$$

In this expression, $k_{0}$ and $\beta$ depend on the slip compliances $\alpha_{(k)}$ and the symmetry of the matrix potential through the relations

$$
k_{0}=\frac{\sum_{k=1}^{K} \alpha_{(k)}\left(1-\cos \left[2\left(\theta_{(k)}-\beta\right)\right]\right)}{\sum_{k=1}^{K} \alpha_{(k)}\left(1+\cos \left[2\left(\theta_{(k)}-\beta\right)\right]\right)}, \quad \tan 2 \beta=\frac{\sum_{k=1}^{K} \alpha_{(k)} \sin 2 \theta_{(k)}}{\sum_{k=1}^{K} \alpha_{(k)} \cos 2 \theta_{(k)}} .
$$

In general, the optimization in equation (2.14) still has to be carried out numerically. However, for macroscopic loadings directed along a slip system (i.e. $\bar{\theta}=\theta_{(k)}$; expression (2.2)), the symmetry of the problem requires the optimal 
$\alpha_{(k)}$ 's to be such that $\beta=\bar{\theta}$. Then, the optimization in equation (2.14) reduces to a one-dimensional minimization with respect to $k_{0}$, which yields $\sqrt{k_{0}}=\left(\sqrt{1+f^{2}}-1\right) / f$, and so the bound $(2.14)$ becomes

$$
\tilde{\tau}_{0}=\sqrt{2} \tau_{0} \frac{1-f}{f}\left(\sqrt{1+f^{2}}-1\right)^{1 / 2},
$$

which is independent of the number of slip systems $K$.

\section{(c) Results and discussion}

This section presents comparisons between the variational $(V A R)$ and relaxed variational $(R V A R)$ bounds of the Hashin-Shtrikman type, as well as the classical Voigt bounds, for the above-described model problem.

Figure 2 provides comparisons among the various bounds for porous, powerlaw materials with square $(K=2)$, hexagonal $(K=3)$ and octagonal $(K=4)$ symmetries, subjected to a macroscopic stress $\overline{\boldsymbol{\sigma}}$ directed along a slip system of the matrix phase $(\bar{\theta}=0)$. In this figure, plots are shown for the effective flow stress $\tilde{\tau}_{0}$, normalized by the flow stress of the matrix $\tau_{0}$, as a function of the strain-rate sensitivity $m$, for a moderate value of the porosity $(f=0.25)$. We begin by noting that, independently of $K$, the VAR and the $R V A R$ bounds coincide for $m=1$ with the linear Hashin-Shtrikman bounds, as they should, and they are seen to improve on the Voigt bound for all values of the strain-rate sensitivity, even though the improvement is less significant in the ideally plastic limit $(m=0)$. The main observation in the context of this figure, however, is that, while the $V A R$ and $R V A R$ bounds agree exactly in the case of a matrix with two slip systems (see part $(a)$ ), the $V A R$ bounds become progressively sharper than the $R V A R$ bounds as the number of slip systems increases, for all values of the strain-rate sensitivity different than 1 (see parts $(c) \&(e)$ ). In fact, in the ideally plastic limit, the RVAR bounds are found to be insensitive to $K$ for this particular loading direction $(\bar{\theta}=0)$, while the corresponding $V A R$ bounds decrease monotonically with increasing $K$. The largest difference between these two sets of bounds is found to be approximately $6 \%$ in the case of $K=4$. Finally, it is noted that the fact that the VAR and $R V A R$ bounds coincide for $K=2$, but not more generally, is because, in that case, the optimal compliances $\hat{\mathbb{S}}_{0}^{(1)}$ associated with both sets of bounds can be written as equation (2.12), with the tensors $\boldsymbol{\mu}_{(k)}$ forming a basis for the relevant (two-dimensional) stress space, so that equality holds in the relaxation of the function $v^{(1)}$ (expression (1.13)).

Also shown in figure 2 are plots for the anisotropy ratio $k_{0}=\lambda_{0} / \mu_{0}$ of the optimal compliances $\hat{\mathbb{S}}_{0}^{(1)}$ associated with the VAR and $R V A R$ bounds. It is recalled that $k_{0}=1$ and 0 correspond to isotropic and strongly anisotropic compliance tensors, respectively. Thus, it is observed that, in general, the optimal $\hat{\mathbb{S}}_{0}^{(1)}$ associated with the VAR bounds tends to be more isotropic as the number of slip systems in the matrix (and therefore the symmetry of the potential $u^{(1)}$ ) increases. In fact, in the case of $K=3$, the potential $u^{(1)}$ is isotropic not only for $m=1$, but also for $m=1 / 3$, and in the case of $K=4$, it is also isotropic for $m=1 / 5$, and so are the optimal $\hat{\mathbb{S}}_{0}^{(1)}$ associated with the corresponding $V A R$ bounds. (Note that the latter are also isotropic for other values of $m$, for which the potential $u^{(1)}$ is anisotropic.) In contrast, the optimal $\hat{\mathbb{S}}_{0}^{(1)}$ associated with the $R V A R$ bounds are always found to be anisotropic for strain-rate sensitivities different than 1 , even if the potential $u^{(1)}$ 
(a)

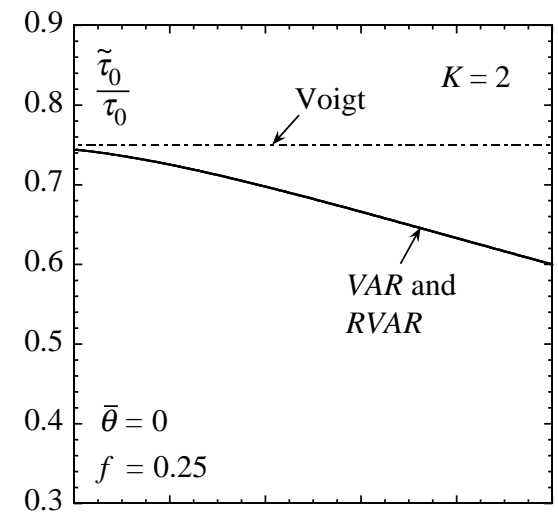

(c)

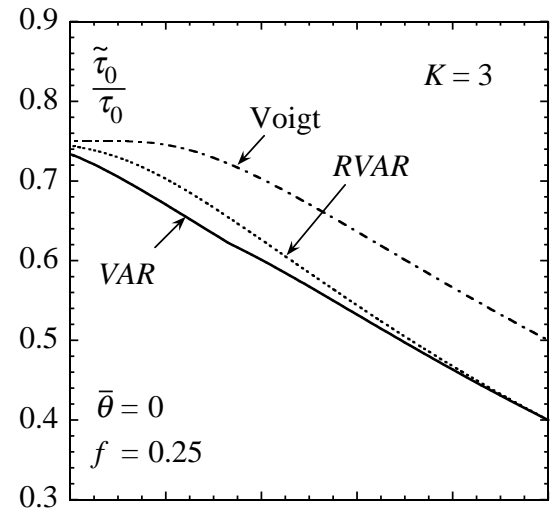

(e)

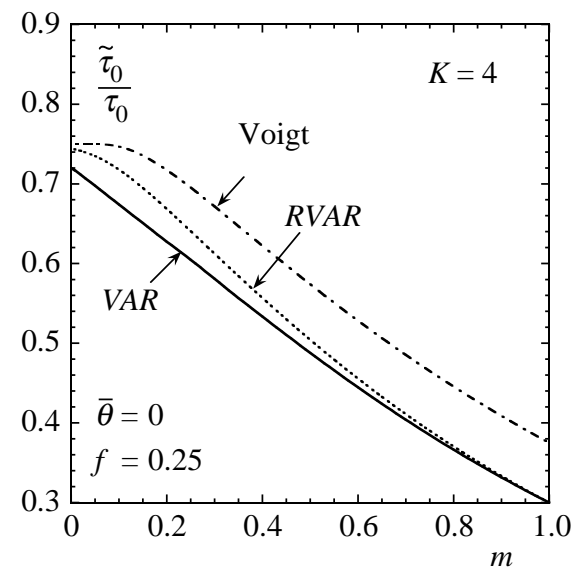

(b)

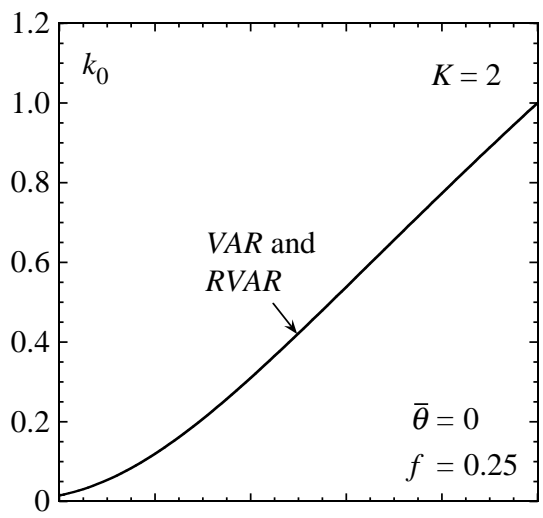

(d)

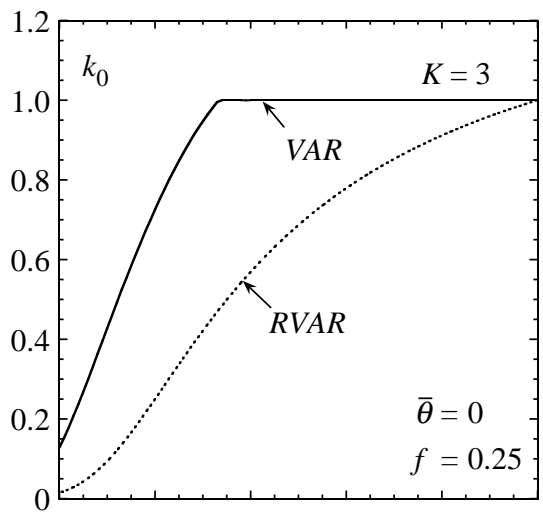

(f)

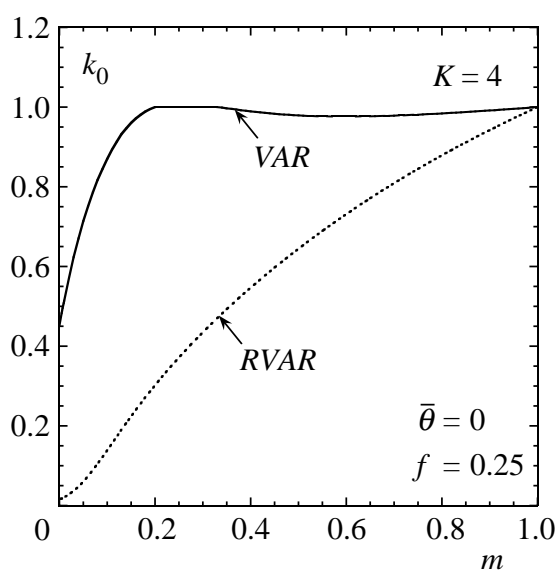

Figure 2. Effective flow stress $\tilde{\tau}_{0}$, normalized by the flow stress of the matrix $\tau_{0}$, and corresponding anisotropy ratios $k$, as a function of the strain-rate sensitivity $m$, for power-law porous materials with square, hexagonal and octagonal symmetry and a given porosity $(f=0.25)$. The macroscopic stress is directed along a slip system $(\bar{\theta}=0)$. Comparisons between the 'variational' $(V A R)$ and 'relaxed variational' $(R V A R)$ bounds of the Hashin-Shtrikman type, and the Voigt bound. 
is isotropic. This is consistent with the fact that, while in the context of the VAR bounds, the tensors $\hat{\mathbb{S}}_{0}^{(r)}$ are identified with secant compliances of the phase potentials, which are known to be isotropic if the potentials are isotropic, in the context of the $R V A R$, the tensors $\hat{\mathbb{S}}_{0}^{(r)}$ are constructed by summing slip secant compliances, and do not correspond to secant compliances of the phase potentials. Finally, it is worth noting that the optimal compliances $\hat{\mathbb{S}}_{0}^{(1)}$ associated with both, the VAR and RVAR bounds of figure 2, have principal axes that are 'aligned' with the symmetry axes of the potential $u^{(1)}$ and the macroscopic stress vector $\overline{\boldsymbol{\sigma}}$ (i.e. $\beta=\bar{\theta}=0$ ), as expected from the symmetry of the problem. It should be emphasized, however, that in the context of the VAR bounds, the optimal orientation of the tensor $\hat{\mathbb{S}}_{0}^{(1)}$ does not follow from a stationarity condition, since the function $v^{(1)}$, as given by equation (1.6), is non-smooth precisely at $\beta=0$.

Figure 3 provides comparisons between the various bounds for the yield surfaces of porous, ideally plastic materials with square $(K=2)$, hexagonal $(K=3)$ and octagonal $(K=4)$ symmetries, for a given value of the porosity $(f=0.25)$. The yield surfaces are symmetric about the $\sigma_{13^{-}}$and $\sigma_{23^{-}}$axes. We begin by noting that, in all three cases, the VAR and $R V A R$ bounds improve on the Voigt bounds for all directions of the macroscopic stress vector $\overline{\boldsymbol{\sigma}}$. The main observation in the context of this figure, however, is that, once again, the VAR and $R V A R$ bounds agree exactly in the case of a matrix phase with two slip systems (see part $(a)$ ), but as the number of slip systems increases, the VAR bounds become progressively sharper than the $R V A R$ bounds, for all directions of the macroscopic stress vector $\overline{\boldsymbol{\sigma}}$ (see parts $(c) \&(e)$ ), the largest difference being of the order of $6 \%$ in the case of a matrix phase with four slip systems. In addition, it is interesting to note that while the macroscopic yield surfaces predicted by the Voigt bounds are simply rescaled versions of the yield surface of the corresponding matrix phase, and therefore exhibit homologous corners, the macroscopic yield surfaces predicted by the VAR and $R V A R$ bounds are smooth and exhibit a more complicated dependence on the loading direction.

Also shown in figure 3 are plots for the anisotropy ratio $k_{0}$ of the optimal $\hat{\mathbb{S}}_{0}^{(1)}$ associated with the VAR and $R V A R$ bounds, as a function of the loading angle $\bar{\theta}$. Given the symmetries of the problem, it is sufficient to restrict attention to values of $\bar{\theta}$ between 0 and $\pi /(2 K)$, which correspond to loadings along a slip system and a corner of the matrix phase, respectively. It can be seen that the optimal $\hat{\mathbb{S}}_{0}^{(1)}$ associated with the $V A R$ bounds are, in general, anisotropic $\left(k_{0}<1\right)$, but become isotropic $\left(k_{0} \rightarrow 1\right)$ as the loading direction approaches that of a corner of the matrix phase $(\bar{\theta} \rightarrow \pi /(2 K))$. On the other hand, the principal axes of $\hat{\mathbb{S}}_{0}^{(1)}$, always in the context of the $V A R$ bounds, remain aligned with the symmetry axes of $u^{(1)}$ (i.e. $\beta=0$ ) for all loading directions in this range. It follows from the symmetry of the problem that, as the loading direction varies from one slip system to an adjacent one, the principal directions of $\hat{\mathbb{S}}_{0}^{(1)}$ 'switch' from one set of symmetry axes of $u^{(1)}$ to another by becoming isotropic in between. Thus, it is found that the principal directions of the optimal $\hat{\mathbb{S}}_{0}^{(1)}$ depend on the direction of loading, as expected, but in such a way that they always coincide with symmetry axes of the phase potential $u^{(1)}$. This fact, if also true in higher dimensions, could be exploited to simplify the computations of the variational bounds in the context of more complex materials with phase potentials exhibiting certain 
(a)

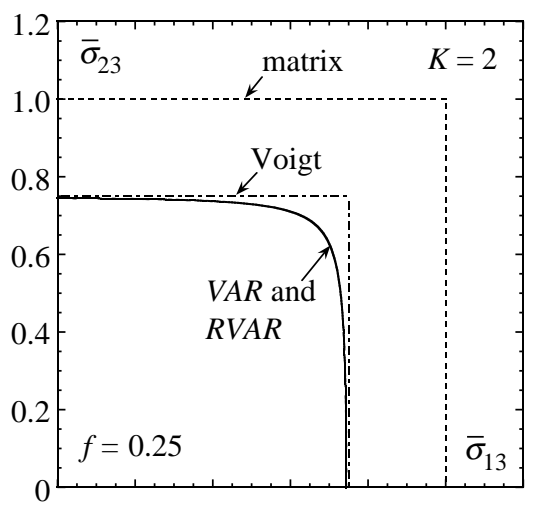

(c)

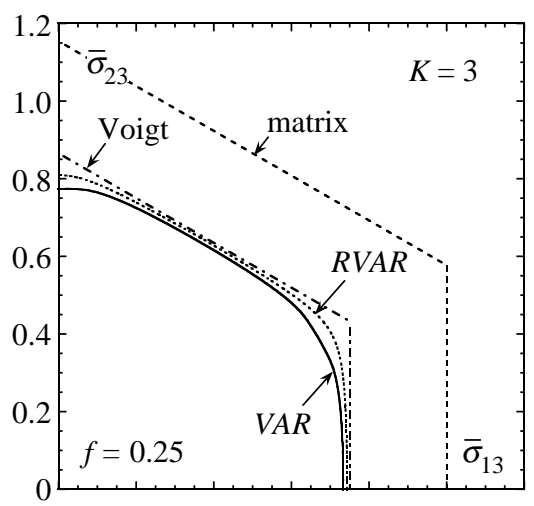

$(e)$

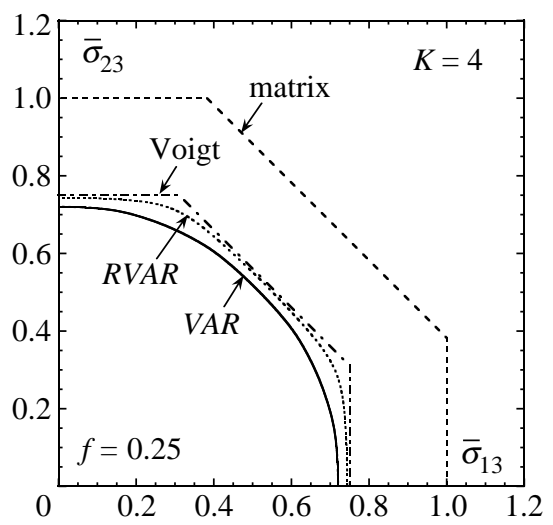

(b)

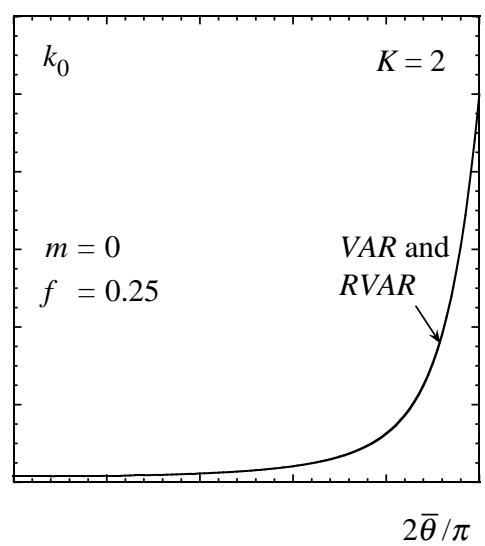

(d)

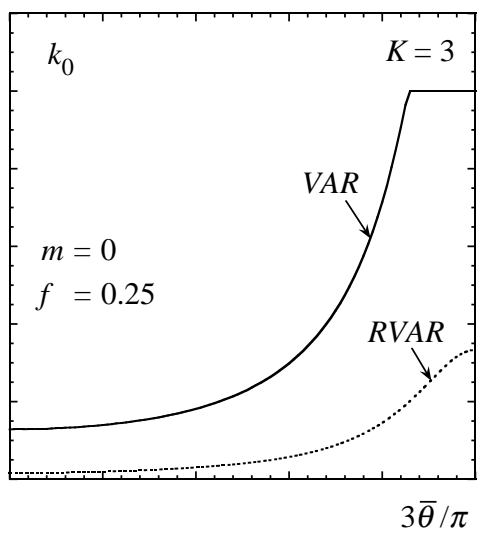

$(f)$

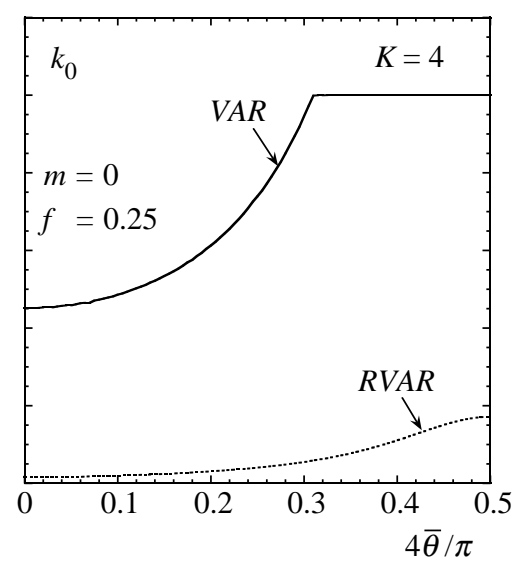

Figure 3. Yield surfaces, normalized by the flow stress of the matrix $\tau_{0}$, and corresponding anisotropy ratios $k_{0}$, for porous, ideally plastic materials with square, hexagonal and octagonal symmetry and a given porosity $(f=0.25)$. Comparisons between the 'variational' $(V A R)$ and 'relaxed variational' (RVAR) bounds of the Hashin-Shtrikman type, and the Voigt bound. 
symmetries, such as polycrystals. In contrast, the optimal compliances $\hat{\mathbb{S}}_{0}^{(1)}$ associated with the $R V A R$ bounds for $K=3$ and 4 are seen to remain anisotropic for all loading directions (see parts $(d) \&(f)$ ), and their principal directions are found to vary smoothly with $\bar{\theta}$, being aligned with the macroscopic stress vector whenever the latter is directed along a symmetry axis of $u^{(1)}$, as expected.

\section{Application to isotropic porous materials}

\section{(a) Power-law porous materials}

It has been found in $\$ 2 c$ that the differences between the variational and relaxed variational bounds become more significant as the number of slip systems in the matrix phase increases. In this section, the limiting case of a power-law matrix phase with an infinite number of slip systems is considered. To that end, it is convenient to consider first the following variant of the matrix potential (1.3) with a finite number of slip systems:

$$
u^{(1)}(\boldsymbol{\sigma})=\frac{2 \pi}{K} \sum_{k=1}^{K} \bar{\psi}\left(\boldsymbol{\sigma} \cdot \boldsymbol{\mu}_{(k)}\right), \quad \bar{\psi}(\tau)=\varrho(n) \frac{\gamma_{0} \tau_{0}}{n+1}\left|\frac{\tau}{\tau_{0}}\right|^{n+1} .
$$

In this expression, the Schmid tensors $\boldsymbol{\mu}_{(k)}$ are given by equation (2.1), with $\boldsymbol{n}_{(k)}$ denoting unit vectors (2.2) normal to the slip planes defined by the angles $\theta_{(k)}=\pi(k-1) / K$, and $\varrho$ is defined as

$$
\varrho(n)=\frac{n+1}{2 n} \frac{\Gamma\left(\frac{n+1}{2}\right)}{\sqrt{\pi} \Gamma\left(\frac{n}{2}\right)},
$$

where $\Gamma$ denotes the Euler gamma function. Then, in the limit $K \rightarrow \infty$, the potential (3.1) becomes

$$
\lim _{K \rightarrow \infty} u^{(1)}(\boldsymbol{\sigma})=\int_{0}^{2 \pi} \bar{\psi}(\boldsymbol{\sigma} \cdot \boldsymbol{\mu}(\theta)) \mathrm{d} \theta=\frac{\tau_{0} \gamma_{0}}{n+1}\left(\frac{\tau_{\mathrm{e}}}{\tau_{0}}\right)^{n+1}=\psi\left(\tau_{\mathrm{e}}\right),
$$

where $\tau_{\mathrm{e}}=\sqrt{(1 / 2) \boldsymbol{\sigma}_{d} \cdot \boldsymbol{\sigma}_{d}}=\left(\sigma_{13}^{2}+\sigma_{23}^{2}\right)^{1 / 2}$ is the equivalent stress. Thus, the potential (3.1) tends to an isotropic, power-law potential $\psi$, as the number of slip systems tends to infinity. In fact, equation (3.3) is nothing more than the planewave decomposition of the isotropic potential $\psi$ (see e.g. Gel'fand \& Shilov 1964). This decomposition makes it possible to apply the relaxed variational method of deBotton \& Ponte Castañeda (1995), which is specifically designed for potentials of the form (1.3), to composites made up of isotropic power-law phases. In addition, it allows us to compare the variational and relaxed variational bounds, in the limiting case of an infinite number of slip systems, where the differences between them are expected to be most significant.

It is recalled that under anti-plane conditions, the effective stress potential $\tilde{u}$ of a porous material with a matrix potential (3.1) can be written as equation (2.3), where the effective flow stress $\tilde{\tau}_{0}$ is a function of the loading direction $\bar{\theta}$, except in the limit $K \rightarrow \infty$, where the material becomes isotropic and, therefore, $\tilde{\tau}_{0}$ independent of $\bar{\theta}$.

Finally, it is recalled that in $\S 2$, only the stress formulations of the variational and the relaxed variational bounds were considered. The reason for this is that the Legendre transform of potentials of the form (1.3) cannot be written as a sum of slip potentials, and therefore a strain-rate version of the relaxed variational 
bounds of deBotton \& Ponte Castañeda (1995) is not available in that case, except for the case of an infinite number of slip systems, when the Legendre transform of equation (3.3), $\psi^{*}$, can in fact be written as an infinite sum of slip potentials, again, by making use of the plane-wave decomposition. This last result will be used below to obtain an alternate relaxed variational bound for porous materials with an isotropic, power-law matrix. On the other hand, there is no point in making use of the dual form (strain-rate version) of the variational bound, since we know from the general theory that it would lead to precisely the same bounds.

\section{(b) Variational bounds}

The variational bounds of the Hashin-Shtrikman type for the effective behaviour of a porous material with a matrix potential (3.1), with finite number of slip systems, can be derived as already discussed in $§ 3 a$. However, in the limiting case of infinitely many slip systems, the matrix potential becomes isotropic, and the optimal compliance tensors $\hat{\mathbb{S}}_{0}^{(1)}$ can be shown to be isotropic, i.e., $\lambda_{0}=\mu_{0}$ in equation (2.5) and $k_{0}=1$. Then, the function $v^{(1)}$ can be easily computed, and the optimization with respect to the single modulus $\mu_{0}$ can be carried out analytically. The resulting variational bound for the effective flow stress is given by

$$
\frac{\tilde{\tau}_{0}}{\tau_{0}}=\frac{1-f}{(1+f)^{(1+m) / 2}} .
$$

This result is in exact agreement with the bound for porous, power-law materials first obtained by Ponte Castañeda (1991) by means of the variational method initially proposed for composites with isotropic phases.

\section{(c) Relaxed variational bounds}

The relaxed variational bounds of the Hashin-Shtrikman type for the effective behaviour of a porous material with a matrix potential (3.1), with finite number of slip systems, are computed in the manner described in $\S 3 b$. Although the matrix potential becomes isotropic for infinitely many slip systems (with the same flow stress), the optimal compliance tensors $\hat{\mathfrak{S}}_{0}^{(1)}$ are found to remain anisotropic (i.e. $k_{0} \neq 1$ ) in this case. But from the symmetry of the problem, it follows that the principal axes of $\hat{\mathbb{S}}_{0}^{(1)}$ should be such that $\beta=\bar{\theta}$. Then, the optimality conditions with respect to the slip compliances simplify, and the relaxed variational bounds for the effective flow stress arising from the stress formulation can be written as

$$
\begin{aligned}
\frac{\tilde{\tau}_{0}}{\tau_{0}}= & \frac{1-f}{\left[1+\frac{f}{2}\left(\sqrt{k_{0}}+\frac{1}{\sqrt{k_{0}}}\right)\right]^{(1+m) / 2}} \\
& \times\left\{\frac{\varrho(n)}{2^{(n+1) / 2}} \int_{0}^{2 \pi}\left|1+\frac{\sqrt{k_{0}}-(f / 2)\left(1-k_{0}\right)}{\sqrt{k_{0}}+(f / 2)\left(1+k_{0}\right)} \cos 2 \theta\right|^{(n+1) / 2} \mathrm{~d} \theta\right\}^{-m},
\end{aligned}
$$


(a)

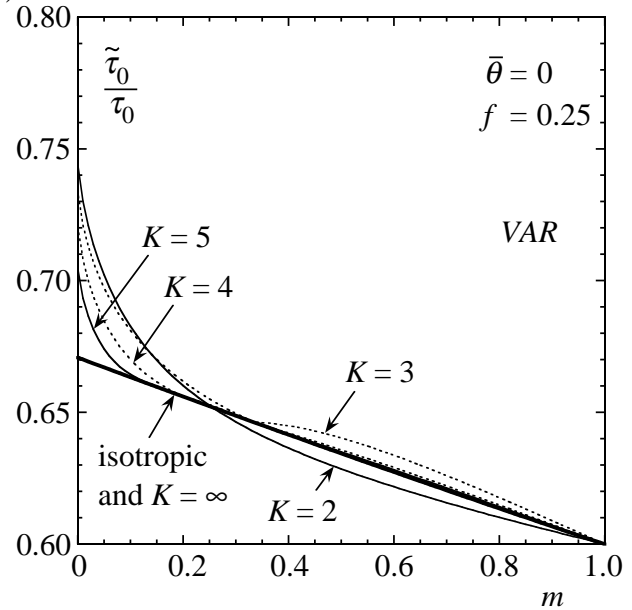

(b)

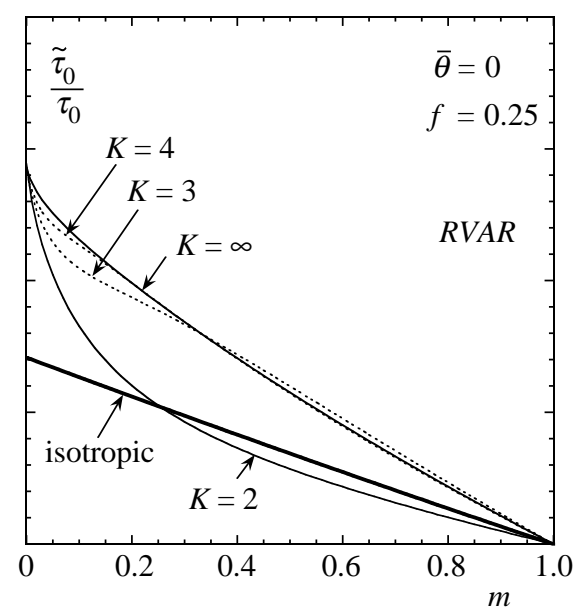

Figure 4. Effective flow stress $\tilde{\tau}_{0}$, normalized by the flow stress of the matrix $\tau_{0}$, of power-law porous materials, as a function of the strain-rate sensitivity $m$, for several numbers of slip systems $K$ and a given porosity $(f=0.25)$. The macroscopic stress is directed along a slip system $(\bar{\theta}=0)$. Comparisons between the $(a)$ 'variational' $(V A R)$ and $(b)$ 'relaxed variational' $(R V A R)$ bounds with the 'variational' bound for isotropic phases.

where $k_{0}$ is the solution to

$$
\int_{0}^{\pi / 2}\left|1+\frac{\sqrt{k_{0}}-(f / 2)\left(1-k_{0}\right)}{\sqrt{k_{0}}+(f / 2)\left(1+k_{0}\right)} \cos 2 \theta\right|^{(n-1) / 2}\left(\cos 2 \theta-\frac{1-k_{0}}{1+k_{0}}\right) \mathrm{d} \theta=0 .
$$

In the ideally plastic limit $(n \rightarrow \infty)$, equation $(3.6)$ yields $\sqrt{k_{0}}=\left(\sqrt{1+f^{2}}-1\right) / f$, and expression (3.5) reduces to equation (2.16). In addition, as already stated above, a dual (strain-rate) version of the bound (3.5) can be obtained in a completely analogous fashion, but it will not be detailed here, for conciseness.

\section{(d) Results and discussion}

In figure 4 , results are provided for porous power-law materials with a matrix potential (3.1), loaded along a slip system $(\bar{\theta}=0)$, for several values of $K$ (number of slip systems). Figure $4 a, b$ shows plots for the stress versions of the variational $(V A R)$ and relaxed variational $(R V A R)$ upper bounds, respectively, for the effective flow stress $\tilde{\tau}_{0}$, normalized by the flow stress of the matrix phase $\tau_{0}$, as a function of the strain-rate sensitivity $m$, for a moderate value of the porosity $(f=0.25)$. Also shown in this figure are the corresponding variational upper bounds for an isotropic matrix, given by equation (3.4). The key observation in the context of this figure is that, while the VAR bounds tend to the 'isotropic' bounds as $K$ tends to infinity, the $R V A R$ bounds tend to a different limit, given by equation (3.5), well above the isotropic bounds, for all values of the strain-rate sensitivity different than 1. In the ideally plastic limit, the difference between the $R V A R$ and the isotropic bounds is of the order of $10 \%$ for this particular value of the porosity.

Direct comparisons between the VAR and RVAR bounds for $K=\infty$ are provided in figure 5 , as a function of the strain-rate sensitivity $m$. Note that both, the stress $(U)$ and the strain-rate $(W)$ versions of the $R V A R$ bounds have been 
(a)

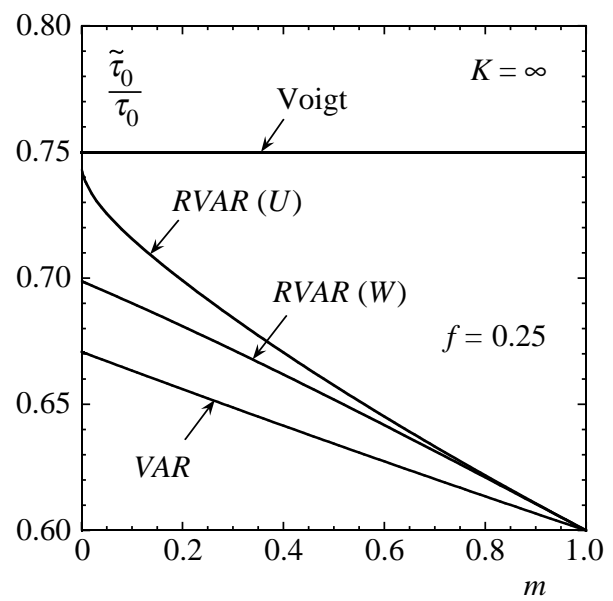

(b)

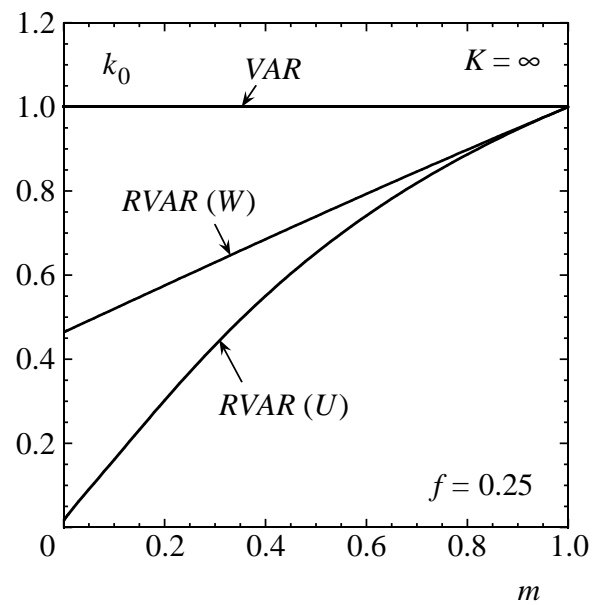

Figure 5 . Effective flow stress $\tilde{\tau}_{0}$, normalized by the flow stress of the matrix $\tau_{0}$, and corresponding anisotropy ratio $k_{0}$, for isotropic power-law porous materials, as a function of the strain-rate sensitivity $m$, for a given porosity $(f=0.25)$. Comparisons between the 'variational' $(V A R)$ and 'relaxed variational' $(R V A R)$ bounds.

included in this figure. The main observation is that, unlike the dual versions of the VAR bounds, the stress and strain-rate versions of the $R V A R$ bounds are not equivalent to each other, for all values of $m$ different than 1 (see part $(a)$ ). In other words, the $R V A R$ bounds exhibit a duality gap, which is seen to increase with increasing nonlinearity. Furthermore, of the two versions of the $R V A R$ bounds, the $R V A R(W)$ bounds are found to be sharper than the $R V A R(U)$ bounds, lying roughly midway between the $V A R$ and $R V A R(U)$ bounds in the ideally plastic limit. The reason for the duality gap in the $R V A R$ bounds is that the functions inside the integrals of the plane-wave decompositions of $\psi$ and $\psi^{*}$ (expression (3.3)) are not Legendre duals of each other, except for $n=1 / m=1$. Finally, part $(b)$ provides plots for the optimal anisotropy ratios $k_{0}$ associated with the bounds shown in part $(a)$. It can be seen that, as already mentioned, the optimal compliance tensor $\hat{\mathbb{S}}_{0}^{(1)}$ associated with the $V A R$ bounds is isotropic (i.e. $\left.k_{0}=1\right)$ for all values of $m$, whereas those associated with the $R V A R(U)$ and $R V A R(W)$ bounds are anisotropic for all values of $m$ different than 1 .

\section{Concluding remarks}

The variational bounds proposed in part I of this work for composites with anisotropic phases have been specialized for composites with crystalline phases and computed for a model (two-phase) porous material with a power-law crystalline matrix phase. It was found that the new variational bounds improve, in general, on the earlier relaxed variational bounds of deBotton \& Ponte Castañeda (1995). The improvement was found to become more significant with increasing nonlinearity and with an increasing number of slip systems, being as much as $10 \%$ in some extreme cases. Although these findings were made in the context of a model (two-phase) system, they are expected to be representative of 
what would happen for more general material systems, including the very important case of polycrystalline aggregates (Dendievel et al. 1991; Willis 1994; deBotton \& Ponte Castañeda 1995). While the computation of variational bounds for general types of viscoplastic polycrystals might be a difficult task due to the non-convex optimizations involved, it should be relatively simple at least in the strongly nonlinear limit of rigid-ideally plastic behaviour, which is actually the most interesting case. Applications to such polycrystalline systems will be pursued in future work. In this context, it should be recalled from part I that bounds have already been obtained for such polycrystalline systems by means of the 'translation' method (Goldstein 2001; Garroni \& Kohn 2003). While thus far these bounds have been computed only for two- and three-dimensional conductivity, they exhibit scaling laws in the anisotropy contrast parameters that are substantially more restrictive than the classical Taylor bound. Owing to this, they will provide useful benchmarks against which to compare the new methodology proposed in this work (when applied to polycrystals).

This material is based upon work supported by the National Science Foundation under Grants CMS-02-01454 and OISE-02-31867.

\section{References}

deBotton, G. \& Ponte Castañeda, P. 1995 Variational estimates for the creep behaviour of polycrystals. Proc. R. Soc. A 448, 121-142. (doi:10.1098/rspa.1995.0009)

Dendievel, R., Bonnet, G. \& Willis, J. R. 1991 Bounds for the creep behaviour of polycrystalline materials. In Inelastic deformation of composite materials (ed. G. J. Dvorak), pp. 175-192. New York, NY: Springer.

Garroni, A. \& Kohn, R. 2003 Some three-dimensional problems related to dielectric breakdown and polycrystal plasticity. Proc. R. Soc. A 459, 2613-2625. (doi:10.1098/rspa.2003.1152)

Gel'fand, I. M. \& Shilov, G. E. 1964 Generalized functions, vol. I. New York, NY: Academic Press.

Goldstein, G. H. 2001 Rigid perfectly plastic two-dimensional polycrystals. Proc. R. Soc. A 457, 2789-2798. (doi:10.1098/rspa.2001.0839)

Idiart, M. I. \& Ponte Castañeda, P. 2007 Variational linear comparison bounds for nonlinear composites with anisotropic phases. I. General results. Proc. R. Soc. A 463, 907-924. (doi:10. 1098/rspa.2006.1797)

Ponte Castañeda, P. 1991 The effective mechanical properties of nonlinear isotropic composites. J. Mech. Phys. Solids 39, 45-71. (doi:10.1016/0022-5096(91)90030-R)

Ponte Castañeda, P. \& Nebozhyn, M. V. 1997 Variational estimates of the self-consistent type for the effective behaviour of some model nonlinear polycrystals. Proc. R. Soc. A 453, 2715-2724.

Ponte Castañeda, P. \& Suquet, P. 1998 Nonlinear composites. Adv. Appl. Mech. 34, 171-302.

Rockafellar, T. 1970 Convex analysis. Princeton, NJ: Princeton University Press.

Storn, R. \& Price, K. 1997 Differential evolution - a simple and efficient heuristic for global optimization over continuous spaces. J. Global Optimization 11, 341-359. (doi:10.1023/ A:1008202821328)

Willis, J. R. 1977 Bounds and self-consistent estimates for the overall properties of anisotropic composites. J. Mech. Phys. Solids 25, 185-202. (doi:10.1016/0022-5096(77)90022-9)

Willis, J. R. 1994 Upper and lower bounds for nonlinear composite behavior. Mater. Sci. Eng. A $175,7-14$. 